\title{
Fresh insight into premature ovarian insufficiency
}

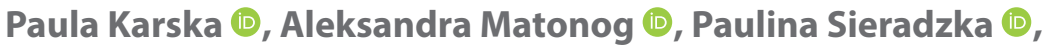 \\ Karolina Kowalczyk (1), Pawel Madej (D) \\ Department of Gynecological Endocrinology, School of Medicine in Katowice, Medical University of Silesia, Katowice, Poland
}

\begin{abstract}
Premature ovarian insufficiency (POI) is one of the vital reasons of anovulatory infertility among women under 40 years old. However, because of the unacknowledged causative factor in most cases, it still remains a huge challenge in gynecology. Recently, the most promising opportunities in diagnosing are connected with the use of some serum biomarkers, such as interleukin-17 (IL-17), Frizzled-5 protein, Soggy-1 protein and other cytokines. Additionally, environmental toxicants such as chemicals and heavy metals might be relevant in the near future when investigating the causes of premature ovarian insufficiency. One of the main aims of the therapy is to focus on maintaining fertility among women with POI, since it is essential for patients considering their young age. Among the newest approaches listed there are different types of stem cells, oocytes donation and in-vitro activation, all of which are recently gaining in importance.

Key words: premature ovarian insufficiency; premature ovarian failure; menopause; cytokines; hormone replacemet therapy; infertility; oncofertility
\end{abstract}

Ginekologia Polska 2021; 92, 7: 518-524

\section{INTRODUCTION}

Premature ovarian insufficiency (POI) is defined as cessation of menstruation before the age of 40 . Principal symptoms include increased levels of follicle stimulating hormone (FSH) and primary or secondary amenorrhea/oligomennorhea prior to the age of 40 . Thus, the condition leads to a hypoestrogenism and decline of residual ovarian follicles [1].

Premature ovarian insufficiency was originally known as premature ovarian failure or premature menopause, but these terms are considered as stigmatizing and suggesting irreversible infertility. In the guidelines of European Society of Human Reproduction and Embryology (ESHRE) from 2015 regarding this issue "premature ovarian insufficiency" was proposed [1]. The following paper includes a literature review of most recent studies published in English in Pubmed/MEDLINE database from January 2014 to December 2020 regarding $\mathrm{POI}$.

\section{EPIDEMIOLOGY}

The prevalence of premature ovarian insufficiency in adults under 40 years old is estimated at approximately $1.1 \%$ of women [2]. Results of cross-sectional multi-ethnic research show that incidence varies depending on ethnicity and is higher among women from Africa and Latin America [1]. The prevalence of POI in Sweden is $1.9 \%$ (90\% of cases are non-iatrogenic), whereas in China it is $2.75 \%[1,3]$. The study conducted in Israel states that the incidence rate of new POI diagnoses per 100,000 person/year doubled (4.5 vs 2.0) during the years 2009-2016, compared with 2000-2008, which may explain the difference in prevalence between past and up-to-date studies [4].

\section{ETIOLOGY}

The etiology of premature ovarian insufficiency is abundant. However, the causative factor in most cases of the syndrome still remains unknown. Chromosomal and genetic defects can cause $\mathrm{POI}$, including chromosome $\mathrm{X}$ defects, fragile-X syndrome, and autosomal monogenic and oligogenic defects. POI may be also associated with autoimmune disorders or infections or have an iatrogenic cause, including surgery, radiotherapy, or chemotherapy. Environmental impact is included in causative factors of POI as well $[5,6]$. Incidence rates of idiopathic $\mathrm{POI}$ have increased by 2.6 -fold and other etiologies by 3.0-fold, while the incidence of genetic disorders such as Turner's syndrome remained constant during the years 2009-2016, compared with 2000-2008 [4].

\footnotetext{
Corresponding author:

Karolina Kowalczyk

Department of Gynecological Endocrinology, School of Medicine in Katowice, Medical University of Silesia, Katowice, Poland

e-mail: karolina.kowalczyk74@gmail.com
}

This article is available in open access under Creative Common Attribution-Non-Commercial-No Derivatives 4.0 International (CC BY-NC-ND 4.0) license, allowing to download articles and share them with others as long as they credit the authors and the publisher, but without permission to change them in any way or use them commercially. 


\section{Genetic causes}

Chromosomal abnormalities and defects can be causation of POI, their frequency is approximately 10-13\% [7]. Chromosome $X$ aneuploidies including: Turner syndrome $(45, X X)$, mosaic forms $(45, X / 46, X X$ and $45, X / 47, X X X)$, trisomy $\mathrm{X}(47, \mathrm{XXX}), \mathrm{X}$-deletions, $\mathrm{X}$-autosomal translocations may lead to premature ovarian insufficiency. Another rearrangement is CGG repeat in the 5 ' regulatory region of the FMR 1 gene, which causes Fragile-X Syndrome [6]. The premutation of FMR1 gene should be investigated in women suffering from POI, because approximately $20-30 \%$ carriers of this expansion will develop fragile X-associated $\mathrm{POI}[6,8]$. What is more, the region from $\mathrm{Xq13.3}$ to $\mathrm{Xq27}$ has been shown to be a critical for normal ovarian function [POI1 (Xq23-Xq27) and POI2 (Xq13-Xq21)], therefore balanced X-autosome translocations or harboring point mutations in this region have been associated with $\mathrm{POI}$ [6].

Due to an expansion of Next Generation Sequencing (NGS), allowing to evaluate numerical changes or genetic defects, there are found many novel genes causing non-syndromic POI, that are summarized in Table 1. There are multiple studies on novel genes associated with premature ovarian insufficiency, however most of them are performed on small sample sizes and restricted to a single ethnic group or even single pedigree line. The cytogenetic, cytogenomic (array comparative genomic hybridization) and exome sequencing approaches have revealed a genetic causation in 20-25\% of POI cases [7].

Summing up, chromosomal analysis should be performed in all patients with non-iatrogenic Premature Ovarian Insufficiency, but autosomal genetic testing is not indicated in women with POI, unless there is an evidence suggesting a specific mutation [5].

\section{Autoimmune causes}

Evidence for an autoimmune etiology is based on the presence of lymphocytic oophoritis, association with other autoimmune disorders, and autoantibodies to ovarian antigens. Isolated lymphocytic oophoritis in POI is rare, occurring only in three percent of patients. Autoimmune oophoritis is characterized by cellular infiltration of the theca cells of growing follicles by macrophages, natural killer cells, T-lymphocytes, plasma cells, and B-lymphocytes. The prime target of the autoimmune attack is the steroid-producing cells, without damaging the primordial and primary follicles.

Approximately $20 \%$ of patients with POI have been already diagnosed with other concomitant autoimmune endocrine diseases like autoimmune thyroid disease (Grave's and Hashimoto's disease), Addison's disease, hypoparathyroidism, hypophysitis, diabetes mellitus type 1 and non-endocrine disorders including chronic candidiasis, idiopathic thrombocytopenic purpura, vitiligo, alopecia,
Table 1. Selection of the novel genes involved in non-syndromic POI etiology classified into groups according to their functions

\begin{tabular}{|c|c|c|}
\hline Genes & Mechanism & References \\
\hline $\begin{array}{l}\text { NANOS3, } \\
\text { EIF4ENIF1, } \\
\text { NOTCH2 }\end{array}$ & Germ cell development & [6] \\
\hline $\begin{array}{l}\text { FSHR, } \\
\text { LHCG, } \\
\text { GJA4 }\end{array}$ & $\begin{array}{l}\text { Oogenesis } \\
\text { and folliculogenesis }\end{array}$ & [6] \\
\hline $\begin{array}{l}\text { NR5A1, } \\
\text { STAR, } \\
\text { ALOX12B }\end{array}$ & Steroidogenesis & {$[6,10]$} \\
\hline $\begin{array}{l}\text { BMP15, BMPR2, } \\
\text { BMPR1A, BMPR1B, } \\
\text { GDF9, } \\
\text { SOHLH1, SOHLH2, } \\
\text { FIGLA, } \\
\text { LHX8, } \\
\text { NOBOX, } \\
\text { ATG7, ATG9A } \\
\text { POLR3A, POLR3B }\end{array}$ & Hormone signaling & {$[6,7,11]$} \\
\hline $\begin{array}{l}\text { MSH4, MSH5, } \\
\text { SPIDR, } \\
\text { FANCM, FANCL, } \\
\text { BNC1, } \\
\text { WDR62, } \\
\text { BRCA2, } \\
\text { TP63, } \\
\text { MCM8, MCM9, } \\
\text { STAG3, } \\
\text { PSMC3IP, } \\
\text { HFM1, } \\
\text { NUP107, } \\
\text { SYCE1, } \\
\text { SYCP2L }\end{array}$ & Meiosis and DNA repair & {$[6,7,9,12]$} \\
\hline $\begin{array}{l}\text { POLR2C, } \\
\text { MRPS22 }\end{array}$ & $\begin{array}{c}\text { Metabolism and protein } \\
\text { synthesis }\end{array}$ & [6] \\
\hline
\end{tabular}

autoimmune hemolytic anemia, pernicious anemia, systemic lupus erythematosus, myasthenia gravis, rheumatoid arthritis, Sjögren's syndrome, primary biliary cirrhosis, chronic active hepatitis, celiac disease and Crohn's disease. Up to $40-50 \%$ of the patients with POI are positive for at least one organ-specific autoantibody [13].

Addison's disease and autoimmune polyendocrine syndrome (APS) type II are known as clinically important factors predisposing to ovarian disorders, therefore affected women should be counseled and screened for POI [5]. APS type II consists of primary adrenal insufficiency, diabetes mellitus type 1, autoimmune thyroid disease, celiac disease and myasthenia gravis [13]. POI of adrenal autoimmune origin is the most frequent type observed in 60 to $80 \%$ of patients with autoimmune cause of ovarian insufficiency. 
Screening for 21-hydroxylase autoantibodies (21OH-Ab) or adrenocortical antibodies (ACA) is recommended in women with POI of an unknown cause or if an autoimmune disorder is suspected [5].

Thyroid autoimmunity is the most prevalent (25-60\%) associated endocrine autoimmune disorder reported in patients with $\mathrm{POI}$ when adrenal autoimmunity is absent [13]. Although untreated hypothyroidism is not life threatening, it can have severe impact on the fetal neurocognitive development in case of pregnancy. Therefore, according to ESHRE guidelines, screening for thyroid antibodies (TPO-Ab) should be performed in women with POI. In patients with a positive TPO-Ab test, thyroid-stimulating hormone (TSH) should be measured every year [5].

\section{latrogenic causes}

Chemotherapy affects the granulosa and theca cells of the ovary more than the oocytes. However, the effect of the chemotherapy depends on the drug (Tab. 2), dose, and the age of the patient [14]. Women under 40 years old develop

Table 2. Chemotherapeutic drugs divided into groups according to their impact on ovarian insufficiency [14]

\begin{tabular}{|l|l|}
\hline $\begin{array}{l}\text { Permanent } \\
\text { hypogonadism }\end{array}$ & $\begin{array}{l}\text { nitrogen mustard, L-phenylalanine mustard, } \\
\text { chlorambucil, cyclophosphamide, melphalan, } \\
\text { busulfan, procarbazine, dacarbazine }\end{array}$ \\
\hline $\begin{array}{l}\text { Likely } \\
\text { ovarian damage }\end{array}$ & $\begin{array}{l}\text { vinblastine, cytosine arabinoside, cis-platinum, } \\
\text { carmustine, lomustine, etoposide, imatinib }\end{array}$ \\
\hline $\begin{array}{l}\text { Small or hardly } \\
\text { any impact }\end{array}$ & $\begin{array}{l}\text { methotrexate, 5-fluorouracil, 6-mercaptopurine, } \\
\text { vincristine, mitomycin }\end{array}$ \\
\hline
\end{tabular}

amenorrhea with high serum gonadotropin concentrations during chemotherapy, but menstruation and fertility may return several months to years after the cessation of the chemotherapy [5, 14].

Unlike cytotoxic therapies, oocytes and ovarian stroma are remarkably sensitive to radiation therapy. Ovarian damage also depends on the dose and on the patient's age. In some studies on the dose of radiation that would cause ovarian insufficiency in $97.5 \%$ of patients, the required dose at birth was $20.3 \mathrm{~Gy}$, at 10 years old $-18.4 \mathrm{~Gy}$, at 20 years old $-16.5 \mathrm{~Gy}$ and at 30 years old $-14.3 \mathrm{~Gy}$. All radiation treatments to the pelvic area will likely result in irreversible ovarian damage [15].

\section{Environmental causes}

Some studies and meta-analysis indicate an association between POI and environmental toxicants $[5,16]$. Environmental causative factors are presented in Figure 1.

\section{DIAGNOSTICS}

\section{Clinical features and diagnostic criteria}

Diagnostic criteria to establish the diagnosis of the premature ovarian insufficiency are based on both presence of menstrual disturbances and biochemical assessments. It applies to women who are less than 40 years old, including patients older than 40 years, but with symptoms onset before the age of 40 .

Therefore, premature ovarian insufficiency should always be excluded in women before 40 with oligomenorrhea/amenorrhea of more than four months duration or estrogen-deficiency symptoms. Primarily, levels of follicle-stimulating hormone (FSH) in serum should be evaluated. The latest recommendations of ESHRE and Polish Society of Reproductive Medicine and Embryology (PTMRiE) proclaim that levels of more than $25 \mathrm{IU} / \mathrm{L}$, obtained twice at
Toxicants increasing apoptosis/atresia of primordial and primary follicles:

- 2-bromopropane (2-BP), cadmium,

- 7,12-dimethylbenz[a]anthracene (DMBA),

- 4-vinylcyclohexene (VCH), triclosan,

- pesticides like methoxychlor (MXC)

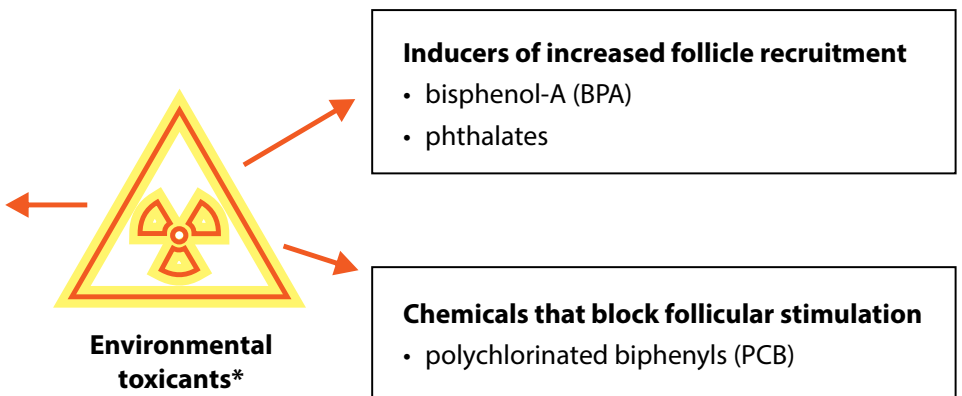

Smoking, alcohol, nutrition, and exposure to endocrine disruptors have been also implicated as influencing the earlier menopause but are not yet established as causes of POI [5].

*For most agents, only experimental data are currently available [16]

Figure 1. Environmental causative factors divided into groups by the mechanism of the ovarian damage 


\begin{tabular}{|c|c|c|}
\hline Protein & $\begin{array}{l}\text { Serum levels in POI patients } \\
\text { compared to healthy control group }\end{array}$ & Characteristic \\
\hline $\begin{array}{l}\text { Interleukin } 17 \text { signaling family } \\
\text { IL-17F } \\
\text { IL-17R } \\
\text { IL-17C }\end{array}$ & $\downarrow$ & $\begin{array}{l}\text { Prognostic factor in ovarian cancer connected } \\
\text { with better prognosis }\end{array}$ \\
\hline Interferon gamma receptor 1 (IFN- $\gamma \mathrm{R} 1)$ & $\downarrow$ & Prognostic factor in ovarian cancer \\
\hline Interleukin 29 & $\downarrow$ & Involved in autoimmune response \\
\hline Neurturin & $\downarrow$ & Follicle maturation and ovulation \\
\hline Frizzled-5 & $\downarrow$ & Receptor for Wnt5a protein - FSH inhibitor \\
\hline Soggy-1 & $\downarrow$ & $\begin{array}{l}\text { Impacts testicular development and spermatogenesis, } \\
\text { but there is poor evidence for role in female fertility }\end{array}$ \\
\hline Heparin Cofactor II (Serpin D1) & $\downarrow$ & $\begin{array}{l}\text { Coagulation factor and a cofactor for heparin } \\
\text { and dermatan sulfate }\end{array}$ \\
\hline Matrix-metalloproteinase -7 (MMP-7) & $\downarrow$ & $\begin{array}{l}\text { Involved in the breakdown of extracellular matrix } \\
\text { during ovulation and menstruation }\end{array}$ \\
\hline Intercellular Adhesion Molecule 3 (ICAM3) & $\downarrow$ & $\begin{array}{l}\text { Plays role in cell adhesion, phagocytosis } \\
\text { and immune response regulation }\end{array}$ \\
\hline Afamin & $\uparrow$ & Marker for oxidative stress \\
\hline
\end{tabular}

least four weeks apart, indicate POI $[5,17]$. Whereas according to previous recommendations the FSH level needed to be greater than $40 \mathrm{IU} / \mathrm{L}$. Anti-Mullerian Hormone (AMH) is an important indicator of diminished ovarian reserve, however, it is not used to establish the diagnosis. Nonetheless, in the absence of those symptoms, the change in regular menstruation in every case should be a reason to do the diagnostic work-up $[18,19]$.

Besides the fact clinicians should enquire about symptoms of estrogen deficiency. Additionally, radiotherapy, surgical interventions on ovaries and cigarette smoking should always raise the doctor's vigilance. Thus, conscientious inquiry into patients' medical history seems to be crucial. Once the diagnosis is established, further assessments need to be done to find a potential cause of the disease.

\section{Serum biomarkers}

The most promising opportunities are connected with the diagnostic use of a serum biomarkers. Jian Liu et al. (2020) suggested that there are many proteins which take part in pathogenesis of premature ovarian insufficiency and may constitute novel biomarkers of this condition [23]. Serum specific $\mathrm{POI}$ proteins profile in patients with $\mathrm{POI}$, healthy fertile women and menopausal women were compared. Interestingly, in each group the array profile of twelve biomarkers was different (Tab. 3).

The level of cytokines from interleukin 17 (IL-17) signaling family (IL-17F, IL-17R, IL-17C) was lower in the serum of $\mathrm{POI}$ patients compared to healthy control group. They are involved not only in immune response, but they are also a crucial part of development of cancers. Elevated level of IL-17 in ovarian cancer is connected with better prognosis, so decreased level in patients with POI can suggest ovarian disease. Another protein, which is a prognostic factor in ovarian cancer is a receptor of Interferon gamma (IFN- $\gamma$ ), IFN- $\gamma$ R1. Its level was also decreased in POI. Overexpression of a dominant-negative mutant of IFN- $\gamma$ R1 lead to a decline in immunogenicity in animal studies and the same mechanism could appear in patient suffering from POI. Downregulation of interleukin 29 (II-29) may be involved in autoimmune response [23].

Decreased level of neurturin protein could be connected with problems with follicle maturation or ovulation, as it is involved in these processes [23].

Furthermore, in POI serum the level of Frizzled- 5 protein was decreased. Frizzled-5 is a receptor for Wnt5a protein. Abedini et al. (2016) [24] emphasized the influence of Wnt5a on women's fertility, because of suppression gonadotropin signaling. Furthermore, Liu et al. [20] suggested that Wnt5a may inhibit FSH due to interaction with its receptor. A decline in amount of Frizzled-5, a receptor for Wnt5a, increases the level of FSH.

There is no evidence that Soggy-1 have a role in female fertility, but there are studies about its impact on testicular development and spermatogenesis, and thus also male fertility. Level of the Soggy-1 protein decreased in women with $\mathrm{POI}$. The results of clinical trial have shown that the level of the Serpin D1 protein, MMP-7 enzyme, Intercellular Adhesion Molecule 3 (ICAM-3) was lower in the serum of $\mathrm{POI}$ patients when compared with healthy control group $[23,26]$. 
Interestingly, afamin protein was the only one marker, which increased in women with POI. Elevated level of afamin occur not only in POI but also in oxidative stress [23].

The clinical trials conducted by Liu et al. have identified several potential premature ovarian insufficiency biomarkers, which can have greater diagnostic value and applicability in clinical practice. Diagnosis of POI based on the detection of genetic abbreviation is extremely expensive and complicated, so these results of papers are promising and give us hope for easier diagnosis. However, Liu et al. [20] emphasize that further studies are needed to confirm their result, because it was the first study, which suggested this association and other studies proving their result should be conducted.

\section{TREATMENT}

Hormone replacement therapy

The British Menopause Society and Women's Health Concern, ESHRE and the American College of Obstetricians and Gynecologists (ACOG) indicate that management of POI should include sex steroid replacement (per os or transdermal)-hormone replacement therapy (HRT) or a combined hormonal contraceptive until the average age of natural menopause is reached, unless they are contraindicated. HRT and combined oral contraceptives are offered to women with POI to decrease risk of breast cancer, cognitive decline, dementia and cardiovascular diseases, to prevent osteoporosis, urogenital atrophy and to improve the quality of a patient's life. Achieving the physiological level of estradiol is the aim of the HRT. Although the frequency of spontaneous pregnancy in women with $\mathrm{POI}$ is low and ranges from 5 to $10 \%$, patients should be advised that the HRT is not any contraceptive. For patients avoiding pregnancy combined hormonal contraceptives are more favorable instead of HRT. Levonorgestrel intrauterine device insertion is recommended for women who want highly effective contraception concurrently preferring some non-contraceptive HRT [21, 22, 27].

$17 \beta$-estradiol, ethinyl estradiol and conjugated equine estrogens can be used in the therapy. However, estradiol is preferred to ethinyl estradiol or conjugated equine estrogens considering bone health and cardiovascular health in women with POI. The delivery of progestogen is required unless the uterus is absent. Oral cyclical micronized natural progesterone is related to the lowest risk of cardiovascular disorders and breast cancer with the endometrial protection compared to the synthetic progestogens [21, 23]. Route of administration depends on the patient ss preferences, however if specific risk factors are present, the transdermal route is preferred [5]. Continuous estrogen replacement is recommended to avoid hypoestrogenism's symptoms. However, cyclical regimens that stimulate active functioning of the endometrium are required for patients desiring pregnancy by oocyte donation, despite the slightly higher risk of the endometrial hyperplasia and carcinoma [22].

Appropriate management of reduced bone mineral density in POI consists of HRT, calcium and/or vitamin $D$ supplementation for women with inadequate vitamin D status and/or calcium intake. Bisphosphonates are not the first-line therapy in women with primary ovarian insufficiency $[22,27]$.

Hypertension, migraine with aura, history of prior venous thromboembolism (VTE), obesity, Turner's syndrome, BRCA1/BRCA2 mutation (without a history of breast cancer after prophylactic bilateral salpingooophorectomy) and fibroids are not contraindications to the HRT, however transdermal delivery of estradiol is preferred. Women with the VTE or thrombophilic disorders should be referred to a hematologist before the HRT prescription.

Hormone replacement therapy is absolutely contraindicated for breast cancer survivors [21, 27]

If there are any contraindications for hormonal treatment, patient should be provided with management of hypoestrogenism's symptoms, cardiovascular diseases prevention and bone protection [21].

Androgen treatment could be also put into consideration for women with POI, however there are limited studies on long-term effects. Androgen replacement therapy is suggested for indications such as memory disorders, neurological complaints, diminished sexual functions and decreased bone density. Evaluation of the therapy is recommended after 2-3 months. Route of administration is similar to the treatment with estrogen and progesterone - depending on the patient's preferences [27].

\section{Non-hormonal therapy}

Cessation of smoking, moderate alcohol intake, balanced diet, maintaining healthy body weight and regular weight-bearing exercises are recommended for the prevention and the therapy of some POI sequelae, especially osteoporosis and cardiovascular disorders [27].

Stem cells therapy is considered to be effective in POI and infertility due to their self-renewal and regeneration potential. There are different types of stem cells used in premature ovarian insufficiency therapy such as mesenchymal stem cells, stem cells from extra-embryonic tissues, induced pluripotent stem cells and ovarian stem cells. Many studies confirmed that stem cell transplants in mice with POI cause a production of ovules and may treat the $\mathrm{POI}$-related infertility [25]. Another approach to the treatment of POI includes the oocytes donation and in vitro activation [29].

Since the diagnosis of POI has a negative impact on emotional and psychological wellbeing, the discussion with the patient is recommended to identify the need for ap- 
propriate psychological support and counseling or other types of therapy provided either on a group or individual basis [21, 27].

\section{FERTILITY AND PREGNANCY}

One of the principal outcomes of premature ovarian insufficiency are fertility problems. There is a small chance of spontaneous pregnancy. Bidet et al. (2011) revealed that $25 \%$ of examined women with idiopathic POI presented features, which indicate resumption of ovarian function. However, pregnancy occurred in only $4.4 \%$ cases $[26,27]$.

Hitherto the solution recommended is in vitro fertilization and embryo transfer (IVF-ET) with the use of donor eggs. Frequently, near relatives donate oocytes, though it may be donated also from unknown donors. However, in group of oocyte donation pregnancies are at high risk of obstetric complications e.g., there is a high prevalence of miscarriage (40\%), in cases of single gestational sac [28].

In vitro activation (IVA) is a novel method, which enables patients suffered from $\mathrm{POI}$ to activation of their residual dormant follicles. Procedure of IVA consist of ovarian cryopreservation, therapy of PTEN (phosphatase and tensin homolog) inhibitor and PI3K (phosphatidylinositol-3-kinase) activator and followed auto-transplantation [28].

The procedure begins with ovariectomy of one or both ovaries, then medulla of ovary is cut into strips. Histological analysis is performed to detect residual follicles. If researcher finds any, the analysis is terminated. Therefore, ovarian strips are fragmented into cubes and treated with a PTEN enzyme inhibitor or a PI3 K stimulator. Next step is the transplantation of ovarian cubes beneath the serosa of one or both Fallopian tubes, this procedure is performed under laparoscopic surgery. This site was chosen, because of high vascularization and convenient monitoring by transvaginal ultrasound and ease for oocyte retrieval for IVF-ET. When preovulatory stage is reached by antral follicles IVF is done $[28,29]$.

The results of studies by Kawamura [28] and Suzuki [29] consist of 37 women, but histological analysis revealed that only 20 of them had residual follicles. Follicle growth was noticed in about $50 \%$ of patients with residual follicles. In some cases, preovulatory stage are detected after six months or longer. IVF and following IVF-ET was done in four patients, but pregnancies were detected in three patients. The outcomes were one miscarriage and two successful deliveries. Endometriosis as a side effect of IVA was detected more frequently in patients with residual follicles and follicle growth.

Suzuki [29] suggested that histological analyses are a better prognosis parameter for predicting in vitro activation success than serum AMH levels. Their results suggest that high level of serum $A M H$ is associated with a better IVA outcome. However, some patients with undetectable AMH levels responded to in vitro activation treatment.

The results have been promising, but future studies are needed to develop a non-invasive method to predict the presence of residual follicles and due to that avoid ovariectomy in several cases. Procedure of in vitro activation could be also a solution for women suffering from other ovarian dysfunction, as well as girls in prepubertal age [28].

Oncofertility is a medical procedure, which aims fertility preservation in women prepared to cancer treatment (radiation or chemotherapy). The choice of appropriate method depends on age of patient, diagnosis and type of treatment. One of the most popular methods is embryo cryopreservation (as a result of in vitro fertilization). This is an appreciated form of preserving fertility, which is gaining popularity, and best if started within 3 days of the onset of cycle. Nevertheless, it is proved that starting the therapy in any moment of the cycle should be equally successful. There is also a possibility to cryopreservation nonfertilized oocytes, recommended in cases, when a patient doesn't have a partner [30, 31].

Ovarian transposition is another method, characterized by surgical repositioning during laparotomy or laparoscopy. It is especially recommended to patients prepared for radiotherapy, because ovaries could be moved well beyond the planned radiation field [30].

\section{CONCLUSIONS}

Premature ovarian insufficiency is a condition of wide-ranging etiology that is expanding even more. Nonetheless, most causative factors are still not established. Fresh approaches of management are deliberated considering not only fertility but also patient's sexuality and novel markers related to the development of premature ovarian insufficiency. The most recent studies suggest that there are some cytokines, which may be novel serum biomarkers for POI. However, it must be confirmed in other studies. Expansion of the Next Generation Sequencing contributes to increasing the number of novel genes associated with POI etiology. In the future sequencing techniques are expected to enable discovering new genetic causes of premature ovarian insufficiency. However, genetic studies should be performed on bigger samples of patients and on different ethnic groups. Currently most studies on novel genes apply only to specific ethnic groups. Therefore, doctors should be aware during counselling patients that some data about genetic causes of POI could be obtained from another ethnic group. Fertility problems are of the most serious difficulties for women with POI. Several approved methods of fertility preservation are available to the patients. However, further research on experimental methods (e.g., in vitro activation) is in progress. 


\section{Conflict of interest}

The authors declare no conflict of interest.

\section{REFERENCES}

1. Rudnicka E, Kruszewska J, Klicka K, et al. Premature ovarian insufficiency - aetiopathology, epidemiology, and diagnostic evaluation. Prz Menopauzalny. 2018; 17(3): 105-108, doi: 10.5114/pm.2018.78550, indexed in Pubmed: 30357004.

2. Wu X, Cai H, Kallianpur A, et al. Impact of premature ovarian failure on mortality and morbidity among Chinese women. PLoS One. 2014; 9(3): e89597, doi: 10.1371/journal.pone.0089597, indexed in Pubmed: 24603759.

3. Lagergren $\mathrm{K}$, Hammar $\mathrm{M}$, Nedstrand $\mathrm{E}$, et al. The prevalence of primary ovarian insufficiency in Sweden; a national register study. BMC Womens Health. 2018; 18(1): 175, doi: 10.1186/s12905-018-0665-2, indexed in Pubmed: 30359245.

4. Gruber N, Kugler S, de Vries L, et al. Primary ovarian insufficiency nationwide incidence rate and etiology among Israeli adolescents. J Adolesc Health. 2020; 66(5): 603-609, doi: 10.1016/j.jadohealth.2019.11.315, indexed in Pubmed: 31987720

5. Webber L, Davies M, Anderson R, et al. European Society for Human Reproduction and Embryology (ESHRE) Guideline Group on POI. ESHRE Guideline: management of women with premature ovarian insufficiency. Hum Reprod. 2016; 31(5): 926-937, doi: 10.1093/humrep/dew027, indexed in Pubmed: 27008889

6. França $M M$, Mendonca BB. Genetics of primary ovarian insufficiency in the next-generation sequencing era. J Endocr Soc. 2020; 4(2): bvz037, doi: 10.1210/jendso/bvz037, indexed in Pubmed: 32099950.

7. Qin Y, Jiao X, Simpson JL, et al. Genetics of primary ovarian insufficiency: new developments and opportunities. Hum Reprod Update. 2015; 21(6): 787-808, doi: 10.1093/humupd/dmv036, indexed in Pubmed: 26243799.

8. Hoyos LR, Thakur M. Fragile $X$ premutation in women: recognizing the health challenges beyond primary ovarian insufficiency. J Assist Reprod Genet. 2017; 34(3): 315-323, doi: 10.1007/s10815-016-0854-6, indexed in Pubmed: 27995424.

9. He WB, Tan C, Zhang YX, et al. Homozygous variants in cause premature ovarian insufficiency. J Med Genet. 2021; 58(3): 168-172, doi: 10.1136/jmedgenet-2019-106789, indexed in Pubmed: 32303603.

10. Alavi A, Darki F, Bidgoli MM, et al. Mutation in ALOX12B likely cause of $\mathrm{POI}$ and also ichthyosis in a large Iranian pedigree. Mol Genet Genomics. 2020; 295(4): 1039-1053, doi: 10.1007/s00438-020-01663-z, indexed in Pubmed: 32253496.

11. Renault L, Patiño LC, Magnin F, et al. BMPR1A and BMPR1B missense mutations cause primary ovarian insufficiency. J Clin Endocrinol Metab. 2020; 105(4): dgz226, doi: 10.1210/clinem/dgz226, indexed in Pubmed: 31769494.

12. Yang $Y$, Guo $T$, Liu $R$, et al. FANCL gene mutations in premature ovarian insufficiency. Hum Mutat. 2020; 41(5): 1033-1041, doi: 10.1002/humu.23997, indexed in Pubmed: 32048394.

13. Domniz N, Meirow D. Premature ovarian insufficiency and autoimmune diseases. Best Pract Res Clin Obstet Gynaecol. 2019; 60: 42-55, doi: 10.1016/j.bpobgyn.2019.07.008, indexed in Pubmed: 31495598.

14. Welt CK, Shapiro CL. Ovarian failure due to anticancer drugs and radiation. UpToDate. https://www.uptodate.com/contents/ovarian-failure-due-to-anticancer-drugs-and-radiation?search=premature ovarian insufficiency\&topicRef $=7435$ \&source $=$ see link\#H465437487 (2020-05-21)

15. Cosgrove $\mathrm{CM}$, Salani R. Ovarian effects of radiation and cytotoxic chemotherapy damage. Best Pract Res Clin Obstet Gynaecol. 2019;
55: 37-48, doi: 10.1016/j.bpobgyn.2018.07.008, indexed in Pubmed: 30166215.

16. Monteiro Cd, Xavier EB, Caetano JP, et al. A critical analysis of the impact of endocrine disruptors as a possible etiology of primary ovarian insufficiency. JBRA Assist Reprod. 2020; 24(3): 324-331, doi: 10.5935/15180557.20200005, indexed in Pubmed: 32202399.

17. Łukaszuk K, Kozioł K, Jakiel G, et al. Diagnostyka i leczenie niepłodności — rekomendacje Polskiego Towarzystwa Medycyny Rozrodu i Embriologii (PTMRiE) oraz Polskiego Towarzystwa Ginekologów i Położników (PTGP). Ginekol i Perinatol Prakt. 2018; 3(3): 112-140.

18. Fraison E, Crawford G, Casper G, et al. Pregnancy following diagnosis of premature ovarian insufficiency: a systematic review. Reprod Biomed Online. 2019;39(3):467-476, doi: 10.1016/j.rbmo.2019.04.019, indexed in Pubmed: 31279714.

19. Michala L, Stefanaki K, Loutradis D. Premature ovarian insufficiency in adolescence: a chance for early diagnosis? Hormones (Athens). 2020; 19(3): 277-283, doi: 10.1007/s42000-019-00141-5, indexed in Pubmed: 31828604

20. Liu J, Huang $X$, Cao $X$, et al. Serum biomarker analysis in patients with premature ovarian insufficiency. Cytokine. 2020; 126: 154876, doi: 10.1016/j.cyto.2019.154876, indexed in Pubmed: 31629109.

21. Hamoda H. British Menopause Society and Women's Health Concern. The British Menopause Society and Women's Health Concern recommendations on the management of women with premature ovarian insufficiency. Post Reprod Health. 2017; 23(1): 22-35, doi: 10.1177/2053369117699358, indexed in Pubmed: 28381102.

22. Committee Opinion No. 698 Summary: Hormone Therapy in Primary Ovarian Insufficiency. Obstet Gynecol. 2017; 129(5): 963-964, doi 10.1097/AOG.0000000000002040, indexed in Pubmed: 28426614.

23. Webber $L$, Anderson RA, Davies M, et al. HRT for women with premature ovarian insufficiency: a comprehensive review. Hum Reprod Open. 2017; 2017(2): hox007, doi: 10.1093/hropen/hox007, indexed in Pubmed: 30895225.

24. Abedini A, Zamberlam G, Lapointe E, et al. WNT5a is required for normal ovarian follicle development and antagonizes gonadotropin responsiveness in granulosa cells by suppressing canonical WNT signaling, FASEB J. 2016; 30(4): 1534-47, https://doi.org/10.1096/ff.15-280313

25. Sheikhansari G, Aghebati-Maleki L, Nouri M, et al. Current approaches for the treatment of premature ovarian failure with stem cell therapy. Biomed Pharmacother. 2018; 102: 254-262, doi: 10.1016/j. biopha.2018.03.056, indexed in Pubmed: 29567538.

26. Bidet $M$, Bachelot $A$, Bissauge $E$, et al. Resumption of ovarian function and pregnancies in 358 patients with premature ovarian failure. J Clin Endocrinol Metab. 2011;96(12): 3864-3872, doi: 10.1210/jc.2011-1038, indexed in Pubmed: 21994953.

27. Guideline of the European Society of Human Reproduction and Embryology. Management of women with premature ovarian insufficiency. POI Guideline Development Group. 2015: 54-62.

28. Kawamura K, Kawamura N, Hsueh AJW. Activation of dormant follicles: a new treatment for premature ovarian failure? Curr Opin Obstet Gynecol. 2016; 28(3): 217-222, doi: 10.1097/GCO.0000000000000268, indexed in Pubmed: 27022685.

29. Suzuki N, Yoshioka N, Takae S, et al. Successful fertility preservation following ovarian tissue vitrification in patients with primary ovarian insufficiency. Hum Reprod. 2015; 30(3):608-615, doi: 10.1093/humrep/deu353, indexed in Pubmed: 25567618.

30. Chan JL, Wang ET. Oncofertility for women with gynecologic malignancies. Gynecol Oncol. 2017; 144(3): 631-636, doi: 10.1016/j. ygyno.2016.12.013, indexed in Pubmed: 28012689.

31. Takai Y. Recent advances in oncofertility care worldwide and in Japan. Reprod Med Biol. 2018; 17(4): 356-368, doi: 10.1002/rmb2.12214, indexed in Pubmed: 30377391. 